policy level, mandatory STI testing and exclusion from work based on a positive test were perceived as largely effective in preventing STIs. However, some participants believed the required panel missed prevalent STIs (e.g. herpes) and modes of transmission (e.g. oral, anal). Unless required by the production company, condom use was rare, and no formal mechanisms existed for notifying partners of an STI. On the economic level, performers' earnings increased with number of sexual partners and riskier sexual acts. Most performers had no health insurance, paying out-of-pocket for STI testing and treatment. Due to the AFI's unsteady payment structure, many performers engaged in sexual escorting to supplement income. On the social level, seasoned performers felt empowered to advocate for their preferences regarding sexual practises and partners on set. However, fear of losing work, compounded by competition among performers, could put newer performers at greater risk for an STI. Although most performers preferred not to use condoms on set, participants described implicit and explicit pressure from producers and directors towards non-condom use.

Conclusion This study highlights important characteristics of the AFI environment that could be targeted for STI prevention interventions.

\section{P3.410 LINK WORKER SCHEME-AN EFFECTIVE RURAL INTERVENTION TO REDUCE THE VULNERABILITY OF CONTRACTING HIV AMONG RURAL SEX WORKERS}

doi:10.1136/sextrans-2013-051184.0862

\section{A Mallick, R Das, A Ghosh. Child In Need Institute, Kolkata, India}

Background Link Worker Scheme, a rural intervention model aims to reach out to the High Risk Groups and vulnerable men and women in rural areas with information, knowledge, skills on STI/ HIV prevention and risk reduction. In the district of Burdwan the programme has reached out to 2629 sex workers to reduce their vulnerability of contracting HIV infection. They are being reached out by the link workers selected from the community itself and they are linked up with the STI clinics and also with government supported Integrated counselling and testing centres for availing services.

Method Since 2009 the programme is being rolled out in 100 villages targeting 2629 rural sex workers. They are made empowered with need based knowledge and skills for reducing their own vulnerability of contracting HIV infection. Side by side various midmedia and awareness programmes are conducted addressing the preventive and vulnerability reduction issues. Condoms are being made available to the sex workers through various channels which are easily accessible to them. After thorough counselling 2080 sex workers have undergone HIV testing at different government supported integrated counselling and testing centres (ICTC). Regular follow-ups are being done by the link workers with support from village level government health service providers and other community stakeholders.

Result From September 2009 to January 2013, 2080 sex workers have undergone HIV testing out of which only $8(0.4 \%)$ have been found HIV positive.

Conclusion Through the programme activities rural sex workers have been reached out effectively by the community based dedicated link workers and the sex workers have realised their own vulnerability and opted for practising safe sexual behaviour in order to reduce their vulnerability of being contracted by the virus. The outcome of the programme has shown a positive response towards achieving the goal of the programme.

\section{P3.411 FACTORS AFFECTING UTILISATION OF STI/HIV CLINICS AMONG SEX WORKERS IN BENIN,}

doi:10.1136/sextrans-2013-051184.0863
'G Batona, ${ }^{2}$ Sémini, 'M Gagnon, ${ }^{3} \mathrm{~F}$ Guedou, 1,4M Alary. 'Université Laval, Québec, OC, Canada; ' World Bank, Washington, WA, United States; ${ }^{3}$ Dispensaire IST, Cotonou, Benin; ${ }^{4} \mathrm{CHU}$ de Québec, Québec, OC, Canada

Background In Benin, HIV/STI clinical and prevention services (based on community mobilisation activities) for female sex worker (FSWs) were extended throughout the country. However, the attendance of clinical services remains very low. Most of the FSW-dedicated clinics are not functional.

Objective This study explores factors related to low functionality and sub-optimal attendance of clinical services by FSWs.

Methods Individual interviews and/or focus groups were conducted with 35 FSWs, 50 health care providers, 70 stakeholders from NGOs and 18 departmental heads of the National AIDS Program in 9 of the 12 regions of the country. Interview guides were developed using a conceptual framework describing actors, implications and issues of implementation activities. Direct observations in the field and analysis of activity reports were also conducted. Data from various sources were triangulated and validated with stakeholders.

Results Several factors have undermined regular STI services utilisation by FSW, encompassing: (i) incomplete and insufficient package of services, with lack of integration of programme components, irregular and insufficient supervision, recurrent shortages of STI drugs and supplies; (ii) limited involvement of FSWs in the programme design, implementation and service organisation; (iii) police repression resulting in dispersion of FSWs, lack of empowerment activities (structural factors); (iv) fear of stigma, little motivation of some health provider to respond to FSWs needs; (v) dysfunctional referral between community level work and STI clinics. In addition poor planning that does not take into account local needs (size, diversification of sex work typologies) also contributes to the non-functionality of the FSW-dedicated clinical services.

Conclusion The results of this study suggest that innovative service delivery models that maximise the synergy between community level work, and uptake of health services need to be designed, implemented and evaluated. Clinical and community level work should also be complemented by appropriate structural interventions.

\section{P3.412 PERSONAL EXPERIENCE OF VIOLENCE AS POTENTIAL RISK FACTOR OF HIV/STI AMONG FSWS IN UKRAINE}

doi:10.1136/sextrans-2013-051184.0864

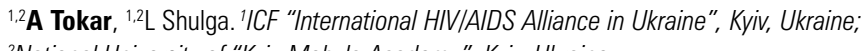
2National University of "Kyiv-Mohyla Academy", Kyiv, Ukraine

Background In Ukraine where the main road of HIV transmission in Ukraine is sexual, female sex workers are considered to have increased risk of HIV and STI infections. Moreover, FSWs suffer from different kinds of violence such as: economic, physical and psychological. The goal of our research was to define if personal experience of violence is associated with risk of HIV/STI infections. Methods Secondary data analysis was performed on the dataset of bio-behavioural survey with 5023 respondents (FSWs) conducted in 2011 by the ICF "HIV/AIDS Alliance in Ukraine". RDS and TLS sampling methodology were applied, only females were recruited. The study was approved by Ethical Review Board of Ukrainian Sociological Association and Institute of epidemiology and infectious diseases named after L. V. Gromashevskyi.

To reveal relationships between HIV/STI rapid tests results and personal experience of violence of FSWs binary logistic regression analysis was provided in SPSS 15.0.

Results About $55 \%$ of respondents were aged 20-30 years old, $52 \%$ - completed secondary, vocational school and obtained not full high education, $64 \%$ - were unmarried and did not live together with sexual partner. Personal experienced of violence were positively 
associated with HIV (OR = 1.8 (1.5-2.1), HCV (OR = 1.7 (1.5-2.0) and syphilis $(\mathrm{OR}=1.5(1.2-1.9)$. Whenever regular partner was source of violence, FSWs had higher chances to be HIV (OR $=1.8$ (1.3-2.6) and HCV (OR = 1.8 (1.3-2.4) positive. Moreover, those who experienced violence from police were more likely to suffer from HIV (OR = 1.4 (1.0-1.8).

Conclusions FSWs, with personal experience of violence, have increased risk of HIV/STI infections. Regular partner as sources of violence is important for HIV and HCV infections, as well.

\section{P3.413 WITHDRAWN BY AUTHOR}

\section{P3.414 ADHERENCE TO HEPATITIS B VACCINE BY FEMALE SEX WORKERS IN A NORTHEAST CITY OF BRAZIL}

doi:10.1136/sextrans-2013-051184.0865

'E Gir, 2R L B Magalhães, 'F M V Pereira, 'R K Reis, 'L P Lopes, ${ }^{3} \mathrm{M} T$ G Galvão, 'S R M S Canini. 'USP - Universidade de São Paulo, Ribeirão Preto/SP, Brazil; '2Universidade Federal do Piauí, Teresina/PI, Brazil; 'Universidade Federal do Ceará, Fortaleza/CE, Brazil

Background Women sex workers are considered vulnerable to sexually transmitted infections, such as hepatitis B and the human immunodeficiency virus (HIV), considering the unfavourable conditions by making verbal agreement with their clients. The study aimed to evaluate the adherence of female sex workers to three doses of hepatitis B vaccine.

Methods Cross-sectional study with female sex workers in the city of Teresina, Brazil. The study included 402 women, using the "snowball" technique. Sociodemographic data were gotten by individual interview, blood sampling for anti-HBs evaluation was collected and the doses of vaccine against hepatitis B were administered, according to the need and acceptance of participants. Data were analysed using Statistical Package for Social Science (SPSS) 18.0.

Results Of the 402 women who participated in study, 96 (23.8\%) reported ever having received at least one dose of the vaccine, while $25(6.2 \%)$ reported having received one dose only, 14 (3.4\%) received two doses, 57 (14.1\%) reported having received three doses and 50 $(12.4 \%)$ did not know. Of those who reported having received three doses was carried anti-HBs, and reagent in 33 (50.7\%). Thus, 57 $(14.1 \%)$ reported already received three doses and 30 (7.4\%) refused to receive the vaccine. Thus, 315 women were vaccinated. Of this total, $223(70.7 \%)$ women receiving two doses and only $92(22.8 \%)$ completed the scheme with three doses of the vaccine.

Conclusion Complete squeme of three doses of the vaccine in this population is challenging, it is necessary for both immunisation strategies and improving access of this population to public health, mainly through primary care.

\section{P3.415 CONCURRENCY \& SEROMIXING AMONG MSM WITH RECENT HIV INFECTION AND NEW HIV DIAGNOSIS: IMPLICATIONS FOR PREP}

doi:10.1136/sextrans-2013-051184.0866

'P M Gorbach, 'H A Pines, 'M Javanbakht, ${ }^{2} \mathrm{R}$ Bolan, ${ }^{1} \mathrm{R}$ E Weiss. 'University of California, Los Angeles, Los Angeles, CA, United States; 'Los Angeles Gay and Lesbian Center, Los Angeles, CA, United States

Background Understanding the frequency of concurrent sexual partnerships, especially with HIV-positive partners, can guide prevention strategies such as pre-exposure prophylaxis (PrEP) for HIVnegative men who have sex with men (MSM).

Methods From 2009-2012, 295 MSM testing for HIV in Los Angeles reported up to 6 male sex partners via computer-assisted selfinterviews.

Concurrency was defined as overlapping dates of sexual partnerships. Nucleic acid amplification tests and detuned assays were performed to determine recent and longstanding HIV infections. Associations between HIV status (recent, longstanding, or negative) and partnership type, concurrency, and serostatus of partners were examined using multinomial logistic regression.

Results Participants were diverse (14.5\% African-American, 27.0\% White, $51.2 \%$ Latino), with mean age of 29.9 years ( $\mathrm{SD}=8.0) ; 42.0 \%$ had recent infection, $27.7 \%$ longstanding infection, and $36.6 \%$ were HIV-negative. $55.5 \%$ reported concurrent partnerships. Of the 887 partnerships reported, $30.6 \%$ (265/866) were main partnerships. Among main partnerships with HIV-negative partners, 47.8\% (77/161) had $\geq 1$ concurrent partner; of those $47.2 \%(34 / 72)$ included $\geq 1 \mathrm{HIV}$-positive/status unknown concurrent partner. In main partnerships with HIV-positive/status unknown partners, $55.1 \%$ (38/69) had $\geq 1$ concurrent partner and of those $65.7 \%$ (23/35) had $\geq 1 \mathrm{HIV}$-positive/status unknown concurrent partner. Adjusting for age and race, compared to HIV-negative testers, having recent infection was associated with substance use (adjusted odds ratio $[\mathrm{AOR}]=1.99,95 \% \mathrm{CI}: 1.03-3.85)$ and concurrency $(\mathrm{AOR}=1.88,95 \% \mathrm{CI}: 1.05-3.39)$; having longstanding infection was associated with no main partner (AOR $=2.27,95 \% \mathrm{CI}: 1.00$ 5.16 ) or $\geq 1 \mathrm{HIV}$-positive/status unknown main partner ( $\mathrm{AOR}=3.63$, 95\% CI: 1.42-9.30) compared to having only HIV-negative main partners, but not with concurrency.

Conclusion The findings associating concurrency with recent infection indicate a broad need for PrEP by MSM with main HIV positive and HIV negative partners. For MSM exposure to HIV may be occurring from concurrent outside partners suggesting a need for PrEP for partnered and non-partnered MSM.

\section{P3.416 HEALTH CARE SEEKING BEHAVIOUR AMONG MALE SEX WORKERS IN SOME SELECTED AREAS}

doi:10.1136/sextrans-2013-051184.0867

1'M Karim, ${ }^{2} \mathrm{~N}$ Mona. ${ }^{1}$ National Institute of Preventive and Social Medicine (NIPSOM), Dhaka, Bangladesh; ${ }^{2}$ Bangladesh Medical college, Dhaka, Bangladesh

Background Male to male sex in Dhaka is fairly widespread and men who make up this group are considered to be vulnerable to contracting and transmitting HIV and other sexually transmitted infections. Men who have unprotected sex with sex workers are at risk not just of contracting HIV and STIs, but of passing them on to their wives and girlfriends.

Methods Cross sectional study was conducted among male sex workers with the objective of assessing their health seeking behaviour. Total 322 sex workers were interviewed by using structured questionnaires.

Results Study revealed that most of the sex workers were young age. Regarding knowledge on how to protect from getting infected with STIs, 90\% respondents had knowledge that STIs can prevented by use condom during sex, $12 \%$ said washing of genitalia with disinfectants after sex, $12 \%$ said by washing with after sex. Regarding STIs majority of the respondents (47\%) perceived that they were at medium risk of getting infected with STIs, $23 \%$ reported at low risk, $13 \%$ reported at high risk and $6 \%$ reported that they were not at all in risk of getting infected with STIs, $12 \%$ respondents thought that they have no idea regarding the risk. Among the respondents $83.3 \%$ suffered ever out of which $66.4 \%$ respondents suffered from STIs once, $27 \%$ twice, $4 \%$ thrice and $4 \%$ more than thrice in the last three months. Thirty seven percent respondents received treatment from NGO clinic, $23 \%$ from MBBS doctor, $17.33 \%$ from government hospital, $13.33 \%$ did self medication, $6 \%$ received treatment from homiopath, $9 \%$ received treatment from kabiraj and $2 \%$ of the respondents did not seek any treatment.

Conclusion It was evident that the knowledge of prevention and treatment measures among the male sex workers was inadequate. 